\title{
Determination of low levels of polycyclic aromatic hydrocarbons in soil by high performance liquid chromatography with tandem fluorescence and diode-array detectors
}

\author{
Yujuan Huang a , Jing Wei ${ }^{\text {a,b }}$, Jing Song ${ }^{\mathrm{a}, \mathrm{b}, *}$, Mengfang Chen ${ }^{\mathrm{a}, \mathrm{b}}$, Yongming Luo ${ }^{\mathrm{a}, \mathrm{b}, \mathrm{c}}$ \\ ${ }^{a}$ Key Laboratory of Soil Environment and Pollution Remediation, Institute of Soil Science, Chinese Academy of Sciences, Nanjing, Jiangsu 210008, China \\ ${ }^{\mathrm{b}}$ Graduate University of Chinese Academy of Sciences, Beijing 100049, China \\ ${ }^{c}$ Yantai Institute of Costal Zone Research, Chinese Academy of Sciences, Yantai, Shandong 264003, China
}

\section{H I G H L I G H T S}

- We set up a method for determination of low levels of 16 PAHs in soil.

- Analyses were performed by HPLC with fluorescence and diode-array detectors.

- The analytical performance of the proposed method were demonstrated.

\section{A R T I C L E I N F O}

\section{Article history:}

Received 31 August 2012

Received in revised form 18 March 2013

Accepted 19 March 2013

Available online 6 May 2013

\section{Keywords:}

HPLC

FLD-DAD

PAHs

Soil

\begin{abstract}
A B S T R A C T
Risk assessment of polycyclic aromatic hydrocarbons (PAHs) contaminated soil and source apportionment require accurate analysis of the concentration of each PAH congener in the soil. However, determination of low level PAH congeners in soil is difficult because of similarity in the chemical properties of 16 PAHs and severe matrix interferences due to complex composition of soils. It is therefore imperative to develop a sensitive and accurate method for determination of low level PAHs in soil. In this work, high performance liquid chromatography equipped with fluorescence and diode-array detectors (HPLCFLD-DAD) was used to determine the concentration of 16 PAHs in soil. The separation of the 16 PAHs was achieved by optimization of the mobile phase gradient elution program and FLD wavelength switching program. Qualitative analysis of the 16 PAHs was based on the retention time (RT) and each PAH specific spectrum obtained from DAD. In contrast, the quantitative analysis of individual PAH congeners was based on the peak areas at the specific wavelength with DAD and FLD. Under optimal conditions the detection limit was in the range $1.0-9.5 \mu \mathrm{g} \mathrm{L}^{-1}$ for 16 PAHs with DAD and $0.01-0.1 \mu \mathrm{g} \mathrm{L}^{-1}$ for $15 \mathrm{PAHs}$ with FLD, and the RSD of PAHs was less than $5 \%$ with DAD and $3 \%$ with FLD. The spiked recoveries were in the range $61-96 \%$, with the exception of $\mathrm{NaP}(<40 \%)$. The results show that HPLC-FLD-DAD can provide more accurate and reliable analysis of low level PAH congeners in soil samples.
\end{abstract}

(c) 2013 Elsevier Ltd. All rights reserved.

\section{Introduction}

Polycyclic aromatic hydrocarbons (PAHs) are a class of diverse organic compounds that typically contain two or more fused aromatic rings. They are ubiquitous environmental pollutants generated primarily during the incomplete combustion of organic materials, in particular fossil fuels such as coal, oil and natural gas, and other hydrocarbons (Lu et al., 2008; Zhang et al., 2011).

\footnotetext{
* Corresponding author at: Key Laboratory of Soil Environment and Pollution Remediation, Institute of Soil Science, Chinese Academy of Sciences, Nanjing, Jiangsu 210008, China. Tel.: +86 25 86881130; fax: +86 2586881126 .

E-mail address: jingsong@issas.ac.cn (J. Song).
}

Due to their carcinogenicity, teratogenicity and mutagenicity (White, 1986), 16 PAHs have been listed by the US Environmental Protection Agency (EPA) as priority pollutants. These are naphthalene, acenaphthene, acenaphthylene, fluorene, phenanthrene, anthracene, fluoranthene, pyrene, benzo[a]anthracne, chrysene, benzo[b]fluoranthene, benzo[k]fluoranthene, benzo[a]pyrene, dibenz[a,h]anthracene, benzo[g,h,i]perylene and indeno[1,2,3-cd]pyrene.

PAHs may enter the soil via wastewater discharge, dry and wet deposition and oil leaks. It has been shown that soil is one of the main sinks for PAHs in the environment (Wilcke, 2000). PAHs that have accumulated in soils may directly or indirectly pose a risk to human and ecosystem health (Jones, 1991). Risk assessment and 
source apportionment of PAH-contaminated soils require accurate analysis of the concentration of each PAH component in the soil. Because of similarity in the chemical properties of 16 PAHs and severe matrix interferences due to complexity of soils, it is imperative to develop a sensitive and accurate method for determination of low level PAHs in soils.

At present the analytical equipment used for the measurement of PAHs in soils mainly comprises gas chromatography (GC) (Kuosmanen et al., 2003; Sikalos and Paleologos, 2005; Zuazagoitia et al., 2009), gas chromatography-mass spectrometry (GC-MS) (Ma et al., 2005; Ozcan et al., 2009; Ene et al., 2012) and high performance liquid chromatography (HPLC) (Chen et al., 2002; Lim et al., 2007; Yang et al., 2011). When GC is used for analysis of PAH components with high boiling points it needs higher temperatures to vaporize, and this may result in a discrimination effect. Moreover, some PAH isomers such as Phenanthrene and Anthracene cannot be easily quantified by GC (Wang et al., 2009). GC-MS equipped with selected ion monitoring (SIM) outperforms GC in PAH isomer separation. However, SIM mode is not useful in further identification of the compound structure, especially when non-target PAH components such as benzo[e]pyrene are present in the sample. In addition, as with GC, GC-MS also needs higher temperatures to vaporize the PAH components with high boiling points (Buco et al., 2004). HPLC is suitable for analysis of compounds with higher molecular weights and boiling points, and has therefore been widely used for PAH analysis. HPLC may be equipped with one of three detectors, namely an ultraviolet (UV), fluorescence (FLD) or diode array detector (DAD). FLD has the characteristics of high sensitivity, high resolution and low detection limits, therefore HPLC-FLD has higher sensitivity for the determination of PAHs exhibiting fluorescent effects. For example, Criado et al. reported that the sensitivity is 4-20 times higher using FLD compared with UV (Criado et al., 2004). However, one main drawback of HPLC-FLD is that the analytes are identified only by their RT. Identification has to be confirmed when samples are complex and many peaks are detected. This can be achieved by using a DAD, which provides the match with specific UV spectra for PAH components (Bouzige et al., 1999). Kicinski et al. used HPLC connected with UV/VIS DAD and FLD to analyze PAHs in drinking water and soil. They concluded that DAD is useful for qualitative and quantitative analysis of PAHs in soil samples and FLD is recommended for the analysis of PAHs in water samples (Kicinski et al., 1989). HPLC-DAD/FLD has been successfully applied in the analysis of PAHs in sewage sludges (Miègea et al., 2003) and food supplements (Danyi et al., 2009). However, the PAHs were detected at a fixed wavelength of $254 \mathrm{~nm}$ with DAD. To our knowledge, there is no report on the use of DAD scanograms to obtain the specific UV spectra of the 16 PAHs for peak identification and peak purity checks, as well as for quantitative analysis of PAHs at each specific UV wavelength.

This present work was aimed at developing a sensitive and reliable method for detection of low level PAHs in soils. Separation of 16 PAHs was achieved by optimizing the mobile phase gradient elution program and the FLD wavelength switching program. The specific UV spectra of the 16 PAHs obtained from DAD were used to confirm the identification of PAH components and to quantitatively analyze PAHs at each specific UV wavelength.

\section{Experimental methods}

\subsection{Apparatus}

Analyses were performed using an HPLC system (Shimadzu, Kyoto, Japan) consisting of a LC-20AT binary pump, a DGU-20A on-line degasser, a SIL-20A autosampler, a CTO-20A column oven,
Table 1

Mobile phase gradient elution program for separation of 16 PAHs.

\begin{tabular}{llll}
\hline Time $(\mathrm{min})$ & Total flow rate $\left(\mathrm{mL} \mathrm{min}^{-1}\right)$ & Acetonitrile $(\%)$ & Water $(\%)$ \\
\hline 0 & 0.5 & 65 & 35 \\
25 & 0.5 & 65 & 35 \\
40 & $\downarrow$ & 90 & 10 \\
60 & 1.0 & $\downarrow$ & $\downarrow$ \\
70 & 1.0 & 100 & 0 \\
\hline
\end{tabular}

a RF-20A fluorescence detector, a SPD-M20A diode array detector and a CBM-20A lite system controller. The data were collected and analyzed using an LC Solution Chromatogram Workstation (Shimadzu, Kyoto, Japan).

\subsection{Reagents}

HPLC grade acetonitrile was obtained from Tedia Company Inc. (Fairfield, $\mathrm{OH}$ ); analytical grade Dichloromethane, n-Hexane, Methanol and Acetone were purchased from Sinopharm Chemical Reagent Co. Ltd. (Shanghai, China); ultra-pure water was prepared daily with a Milli-Q water purification system (Millipore, Billerica, MA). All other reagents used were of analytical grade quality.

\subsection{Standard solution}

A standard mixture of the 16 PAHs $\left(100.0 \mathrm{mg} \mathrm{L}^{-1}\right)$ was purchased from Dr. Ehrenstorfer GmbH (Augsburg, Germany), comprising Naphthalene (NaP), Acenaphthylene (AcPy), Acenaphthene (Ace), Fluorene (Flu), Phenanthrene (Phe), Anthracene (AnT), Fluoranthene (F1uA), Pyrene (Pyr), Benzo(a)anthracene (BaA), Chrysene (Chry), Benzo(b)fluoranthene (BbF), Benzo(k)fluoranthene (BkF), Benzo(a)pyrene (BaP), Dibenzo(a,h)acenaphthene (DBA), Benzo(a) [ghi]perylene (BghiP), and Indeo[1,2,3-cd] pyrene acenaphthene (In-[1,2,3-cd]P) congener.

A $1.0 \mathrm{mg} \mathrm{L}^{-1}$ mixture of the 16-PAH stock solution was obtained by diluting the standard solution with acetonitrile, charging into ampoules and then sealing. All stock and standard solutions were stored at $4{ }^{\circ} \mathrm{C}$. All working solutions were prepared immediately before the experiment by diluting the stock solution.

\subsection{Soil sample preparation}

\subsubsection{Sample extraction}

$2.00-\mathrm{g}$ aliquots of dried and homogenized soils sieved through $0.15 \mathrm{~mm}$ mesh were extracted in a Soxhlet extraction system with $65 \mathrm{~mL}$ of mixed $\mathrm{n}$-hexane/acetone solvent $(1: 1, \mathrm{v} / \mathrm{v})$ for $24 \mathrm{~h}$. The resulting crude extracts were evaporated to dryness using a Model 850 rotary evaporator (Büchi, Flawil, Switzerland) with the water bath at $40^{\circ} \mathrm{C}$, a pressure of $500 \mathrm{mbar}$ and a rotation rate of

Table 2

Wavelength switching program for FLD.

\begin{tabular}{|c|c|c|c|}
\hline $\begin{array}{l}\text { Time } \\
(\mathrm{min})\end{array}$ & $\begin{array}{l}\lambda_{\mathrm{ex}} \\
(\mathrm{nm})\end{array}$ & $\begin{array}{l}\lambda_{\mathrm{em}} \\
(\mathrm{nm})\end{array}$ & PAHs determined \\
\hline 0.01 & 270 & 323 & $\begin{array}{l}\text { 1. NaP; 2. AcPy (no fluorescence); 3. Ace; } 4 . \\
\text { Flu }\end{array}$ \\
\hline 24.1 & 252 & 370 & 5. $\mathrm{PhA}$ \\
\hline 27.0 & 252 & 402 & 6. AnT \\
\hline 30.0 & 280 & 460 & 7. FluA \\
\hline 33.0 & 270 & 390 & 8. Pyr; 9. BaA; 10. Chry \\
\hline 42.5 & 290 & 410 & $\begin{array}{l}\text { 11. BbF; 12. BkF; 13. BaP; 14. DbA; } 15 . \\
\text { BghiP }\end{array}$ \\
\hline 60.0 & 290 & 500 & 16. In-[1, 2, 3-cd]P \\
\hline
\end{tabular}


$35 \mathrm{rev} \mathrm{min}^{-1}$. The extract was then re-dissolved in $2.0 \mathrm{~mL}$ cyclohexane.

\subsubsection{Sample purification}

The re-dissolved extracts $(0.5 \mathrm{~mL})$ were purified by silica gel column chromatography. Each extract was loaded onto the column which was eluted with acetone/n-hexane $(1: 1, v / v)$. The first $1.0 \mathrm{~mL}$ of eluate was discarded and then $2.0 \mathrm{~mL}$ of eluate were collected containing PAH components. Each eluate was dried to remove the solvent with a stream of nitrogen at a flow rate of 3-4 $\mathrm{mL} \mathrm{min}^{-1}$ in a water bath at $40{ }^{\circ} \mathrm{C}$. Then acetonitrile was added to re-dissolve the extracts to give a constant volume of $2.0 \mathrm{~mL}$. The resulting extracts were analyzed using the HPLC-FLD-DAD system.

\subsection{Analytical conditions}

Separation of the 16 PAHs was performed with a $4.6 \times 250 \mathrm{~mm}$ analytical column (Pursuit 5 PAH, Varian, Middelburg, the Netherlands) combined with a $3 \times 10 \mathrm{~mm}$ pre-column (Pursuit PAH) at a constant temperature of $31^{\circ} \mathrm{C}$. The mobile phases are made of acetonitrile and water in different ratios, and the gradient elution program is featured by changing ratios of acetonitrile and water as well as total flow rate at specified time (Table 1). Notably, the total
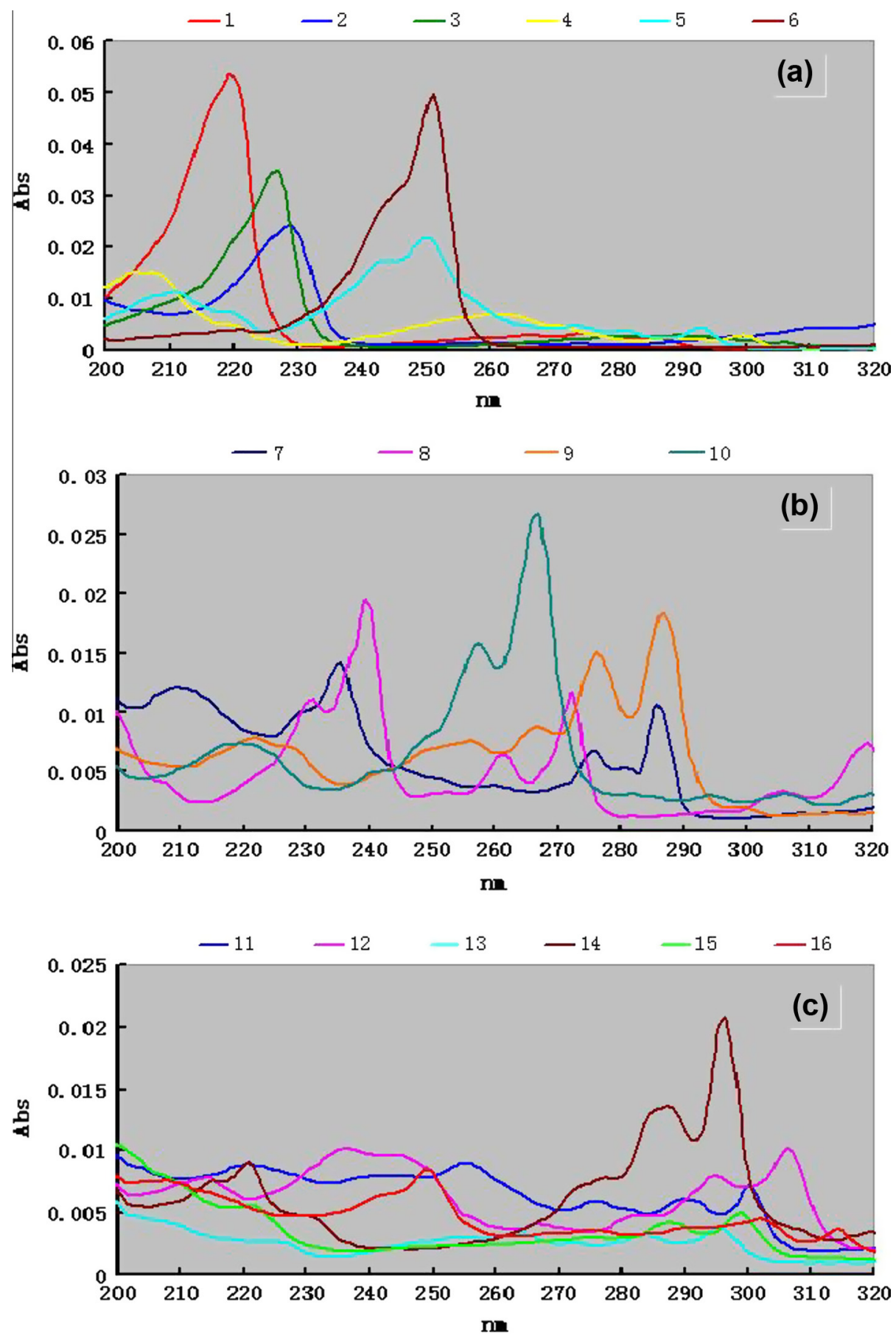

Fig. 1. Specific UV spectra of the 16 PAHs by DAD. 


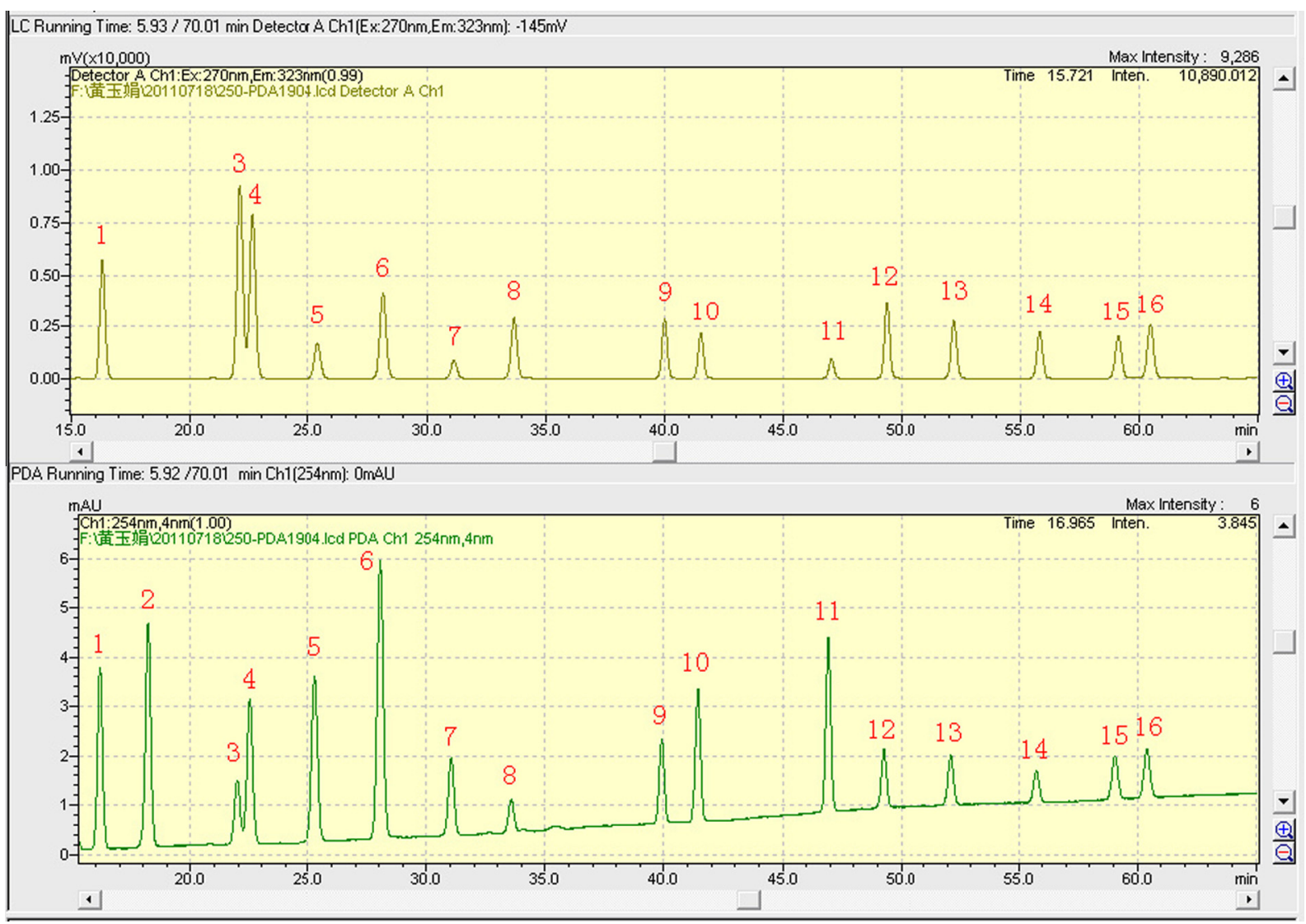

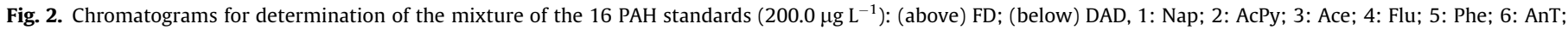
7:FluA; 8: Pyr; 9: BaA; 10: Chry; 11: BbF; 12: BkF; 13: Bap; 14: DahA; 15: BghiP; and 16: In[1,2,3-cd]P.

Table 3

Linearity and detection limit (DL) for the determination of 16 PAHs by HPLC-FLD-DAD.

\begin{tabular}{|c|c|c|c|c|c|c|}
\hline \multirow[t]{2}{*}{$\mathrm{PAH}$} & \multicolumn{3}{|l|}{ FLD } & \multicolumn{3}{|l|}{ DAD } \\
\hline & Regression equation $^{\mathrm{a}}$ & $R^{2}$ & $\mathrm{DL}\left(\mu \mathrm{g} \mathrm{L}^{-1}\right)$ & Regression equation $^{\mathrm{a}}$ & $R^{2}$ & $\mathrm{DL}\left(\mu \mathrm{g} \mathrm{L}^{-1}\right)$ \\
\hline $\mathrm{NaP}$ & $y=41110 x+151717$ & 0.9994 & 0.08 & $y=763.44 x+2312.6$ & 0.9995 & 1.2 \\
\hline AcPy & - & - & - & $y=419.5 x+392.34$ & 0.9999 & 2.3 \\
\hline Ace & $y=69316 x+258493$ & 0.9997 & 0.05 & $y=622.79 x+292.75$ & 0.9999 & 1.8 \\
\hline Flu & $y=307352 x+472205$ & 0.9998 & 0.01 & $y=133.93 x-13$ & 0.9999 & 7.3 \\
\hline Phe & $y=133287 x+331173$ & 0.9994 & 0.02 & $y=426.01 x+172.56$ & 0.9999 & 2.4 \\
\hline AnT & $y=399746 x+444190$ & 0.9998 & 0.01 & $y=964.48 x+554.17$ & 0.9999 & 1.0 \\
\hline FluA & $y=33823 x+19615$ & 0.9999 & 0.10 & $y=255.31 x+390.35$ & 0.9999 & 3.8 \\
\hline Pyr & $y=199955 x+216075$ & 0.9998 & 0.02 & $y=318.45 x+142.78$ & 0.9999 & 3.1 \\
\hline $\mathrm{BaA}$ & $y=189229 x+170269$ & 0.9999 & 0.02 & $y=318.7 x+293.13$ & 0.9999 & 2.7 \\
\hline Chry & $y=149269 x+129813$ & 0.9998 & 0.02 & $y=399 x+302.55$ & 0.9999 & 2.2 \\
\hline $\mathrm{BbF}$ & $y=30856 x+24254$ & 0.9999 & 0.09 & $y=147.15 x+37.35$ & 0.9999 & 5.2 \\
\hline $\mathrm{BkF}$ & $y=242440 x+228782$ & 0.9999 & 0.01 & $y=183.02 x+84.89$ & 0.9999 & 4.8 \\
\hline $\mathrm{BaP}$ & $y=207573 x+59442$ & 0.9999 & 0.01 & $y=110.06 x-132.08$ & 0.9997 & 8.2 \\
\hline DahA & $y=80948 x+79088$ & 0.9999 & 0.04 & $y=304.64 x+286.15$ & 0.9999 & 2.9 \\
\hline Bghi P & $y=72082 x+73728$ & 0.9999 & 0.05 & $y=98.48 x-67.38$ & 0.9998 & 9.5 \\
\hline $\operatorname{In}[1,2,3-\mathrm{cd}] \mathrm{P}$ & $y=223577 x+183312$ & 0.9999 & 0.02 & $y=152.17 x+9.84$ & 0.9998 & 6.5 \\
\hline
\end{tabular}

a $y$, peak area; $x$, PAH concentration $\left(\mu \mathrm{g} \mathrm{L}^{-1}\right)$

flow rate was changing linearly from $0.5 \mathrm{~mL} \mathrm{~min}^{-1}$ at $25 \mathrm{~min}$ to $1.0 \mathrm{~mL} \mathrm{~min}^{-1}$ at $60 \mathrm{~min}$ and the percentage of acetonitrile was changing linearly from $65 \%$ at $25 \mathrm{~min}$ to $90 \%$ at $40 \mathrm{~min}$ and to $100 \%$ at $70 \mathrm{~min}$. The sample injection volume was $10 \mu \mathrm{L}$. The FLD was in medium sensitivity mode with response $1.0 \mathrm{~s}$, and gain $\times 4$. The PAH spectra were recorded by DAD within the range 190$800 \mathrm{~nm}$ with a slit width of $1.2 \mathrm{~nm}$.

\section{Results and discussion}

\subsection{FLD wavelength switching program}

To achieve the highest sensitivity for PAH analysis, the FLD was time programmed for the best excitation and emission wavelengths $\left(\lambda_{\text {ex }} / \lambda_{\text {em }}\right)$ for groups of successively eluting PAHs with 
Table 4

Recovery of the proposed method.

\begin{tabular}{|c|c|c|c|c|c|c|}
\hline \multirow[t]{2}{*}{$\mathrm{PAH}$} & \multirow{2}{*}{$\begin{array}{l}\text { Background value } \\
\left(\mu \mathrm{g} \mathrm{L}^{-1}\right)\end{array}$} & \multirow{2}{*}{$\begin{array}{l}\text { Added concentration } \\
\left(\mu \mathrm{g} \mathrm{L}^{-1}\right)\end{array}$} & \multirow{2}{*}{$\begin{array}{l}\text { FLD } \\
\text { Measured concentration } \\
\left(\mu \mathrm{g} \mathrm{L}^{-1}\right)\end{array}$} & \multicolumn{3}{|l|}{ DAD } \\
\hline & & & & $\begin{array}{l}\text { Recovery rate } \\
(\%)\end{array}$ & $\begin{array}{l}\text { Measured concentration } \\
\left(\mu \mathrm{g} \mathrm{L}^{-1}\right)\end{array}$ & $\begin{array}{l}\text { Recovery rate } \\
(\%)\end{array}$ \\
\hline \multirow[t]{3}{*}{$\mathrm{NaP}$} & 6.9 & 37.5 & 8.2 & 18.4 & 15.9 & 35.8 \\
\hline & 6.9 & 75.0 & 14.1 & 17.2 & 26.8 & 32.7 \\
\hline & 6.9 & 115.5 & 46.3 & 37.8 & 37.4 & 29.8 \\
\hline \multirow[t]{3}{*}{ AcPy } & 141.4 & 37.5 & - & - & 114.9 & 64.2 \\
\hline & 141.4 & 75.0 & - & - & 146.1 & 67.5 \\
\hline & 141.4 & 115.5 & - & - & 162.4 & 63.2 \\
\hline \multirow[t]{3}{*}{ Ace } & 86.2 & 37.5 & 75.2 & 60.8 & 78.3 & 63.2 \\
\hline & 86.2 & 75.0 & 98.7 & 61.2 & 97.9 & 60.7 \\
\hline & 86.2 & 115.5 & 127.4 & 63.2 & 123.6 & 61.3 \\
\hline \multirow[t]{3}{*}{ Flu } & 53.2 & 37.5 & 55.4 & 61.1 & 57.7 & 63.6 \\
\hline & 53.2 & 75.0 & 85.5 & 66.7 & 89.4 & 69.8 \\
\hline & 53.2 & 115.5 & 105.2 & 62.4 & 117.5 & 69.6 \\
\hline \multirow[t]{3}{*}{ Phe } & 143.8 & 37.5 & 160.5 & 88.5 & 152.6 & 84.1 \\
\hline & 143.8 & 75.0 & 199.2 & 91.0 & 198.8 & 90.9 \\
\hline & 143.8 & 115.5 & 208.2 & 80.3 & 204.7 & 78.9 \\
\hline \multirow[t]{3}{*}{ AnT } & 21.4 & 37.5 & 46.7 & 79.4 & 44.6 & 75.7 \\
\hline & 21.4 & 75.0 & 61.0 & 63.2 & 61.2 & 63.5 \\
\hline & 21.4 & 115.5 & 83.5 & 60.9 & 87.3 & 63.7 \\
\hline \multirow[t]{3}{*}{ FluA } & 37.7 & 37.5 & 77.2 & 102.6 & 65.8 & 87.5 \\
\hline & 37.7 & 75.0 & 102.1 & 90.6 & 95.9 & 85.1 \\
\hline & 37.7 & 115.5 & 127.4 & 83.2 & 120.7 & 78.8 \\
\hline \multirow[t]{3}{*}{ Pyr } & 69.9 & 37.5 & 101.5 & 94.5 & 100.5 & 93.5 \\
\hline & 69.9 & 75.0 & 128.9 & 88.9 & 128.7 & 88.9 \\
\hline & 69.9 & 115.5 & 154.7 & 83.4 & 155.6 & 83.9 \\
\hline \multirow[t]{3}{*}{$\mathrm{BaA}$} & 74.5 & 37.5 & 105.9 & 94.5 & 103.0 & 91.9 \\
\hline & 74.5 & 75.0 & 127.8 & 85.5 & 125.8 & 84.2 \\
\hline & 74.5 & 115.5 & 155.9 & 82.1 & 152.2 & 80.1 \\
\hline \multirow[t]{3}{*}{ Chry } & 82.9 & 37.5 & 117.2 & 97.4 & 109.4 & 90.9 \\
\hline & 82.9 & 75.0 & 142.4 & 90.2 & 138.4 & 87.6 \\
\hline & 82.9 & 115.5 & 170.6 & 86 & 166.8 & 84.1 \\
\hline \multirow[t]{3}{*}{$\mathrm{BbF}$} & 81.6 & 37.5 & 110.9 & 93.1 & 107.8 & 90.5 \\
\hline & 81.6 & 75.0 & 139.8 & 89.3 & 134.2 & 85.7 \\
\hline & 81.6 & 115.5 & 168.6 & 85.5 & 161.1 & 81.7 \\
\hline \multirow[t]{3}{*}{$\mathrm{BkF}$} & 53.3 & 37.5 & 84.7 & 93.3 & 84.9 & 93.5 \\
\hline & 53.3 & 75.0 & 112.6 & 87.8 & 111.9 & 87.2 \\
\hline & 53.3 & 115.5 & 140.1 & 83 & 139.5 & 82.6 \\
\hline \multirow[t]{3}{*}{$\mathrm{BaP}$} & 13.3 & 37.5 & 39.1 & 76.9 & 38.9 & 76.5 \\
\hline & 13.3 & 75.0 & 57.3 & 64.9 & 58.0 & 65.7 \\
\hline & 13.3 & 115.5 & 80.9 & 62.8 & 83.0 & 64.4 \\
\hline \multirow[t]{3}{*}{ DahA } & 94.3 & 37.5 & 125.8 & 95.5 & 122.9 & 93.2 \\
\hline & 94.3 & 75.0 & 153.8 & 90.9 & 152.3 & 90.0 \\
\hline & 94.3 & 115.5 & 181.2 & 86.4 & 179.9 & 85.8 \\
\hline \multirow[t]{3}{*}{ Bghi P } & 129.9 & 37.5 & 161.7 & 96.6 & 160.8 & 96.0 \\
\hline & 129.9 & 75.0 & 192.3 & 93.9 & 190.7 & 93.1 \\
\hline & 129.9 & 115.5 & 217.4 & 88.6 & 220.6 & 89.9 \\
\hline \multirow{3}{*}{$\begin{array}{l}\operatorname{In}[1,2,3- \\
\quad \text { cd }] P\end{array}$} & 84.7 & 37.5 & 115 & 94.1 & 106.6 & 87.2 \\
\hline & 84.7 & 75.0 & 138.7 & 86.8 & 132.2 & 82.8 \\
\hline & 84.7 & 115.5 & 165.2 & 82.5 & 158.3 & 79.1 \\
\hline
\end{tabular}

similar spectroscopic characteristics (Kicinski et al., 1989). At $254 \mathrm{~nm}$ of the DAD spectrogram, all 16 PAHs had absorption peaks and were completely separated from each other. The time programmed wavelength switching for FLD was established based on the RT and the $\lambda_{\mathrm{ex}} / \lambda_{\mathrm{em}}$ of the individual PAHs (Table 2). In this time program the $16 \mathrm{PAH}$ peaks had base line separation and there was adequate time between the peaks for all the operations which accompanied the wavelength switching.

\subsection{Selection of quantitative wavelength for $D A D$}

Many PAHs possess very specific UV spectra (Bouzige et al., 1999). Although most PAHs have absorption at $254 \mathrm{~nm}$, this is not the specific UV wavelength for all PAH components. Only by measurement at specific UV wavelength can the highest sensitivity and lowest possible detection limit be achieved. In the present study DAD provided the match with UV spectra for the 16 PAHs by scanning from 190 to $800 \mathrm{~nm}$ (Fig. 1). This shows that the absorptivity of PAHs varies at different UV wavelengths. For instance, at $295 \mathrm{~nm}$ (C-14) DahA has a sharp absorbtion peak but Phe shows little or no absorption at this wavelength and has a sharp absorption peak at $251 \mathrm{~nm}$ (A-5). Selection of the specific UV wavelength as the quantitative wavelength (Table S1, SD) can achieve the highest sensitivity for determination of PAHs. For example, the detection limit of AcPy was $2.3 \mu \mathrm{g} \mathrm{L} \mathrm{L}^{-1}$ at $229 \mathrm{~nm}$ in this study, which was lower than $30.0 \mu \mathrm{g} \mathrm{L}{ }^{-1}$ at $254 \mathrm{~nm}$ reported by Rao et al. (Rao et al., 2007) and its lowest detection limit $\left(2.3 \mu \mathrm{g} \mathrm{L}^{-1}\right)$ at $229 \mathrm{~nm}$ in this study.

\subsection{Analytical performance}

To understand the analytical performance of the proposed method, a series of the 16-PAH working solutions with concentrations of $0.125,0.50,1.25,5.0,10.0,25.0,50.0,100.0,200.0,400.0$ or $800.0 \mu \mathrm{g} \mathrm{L}^{-1}$ were prepared. Under the optimal conditions two 


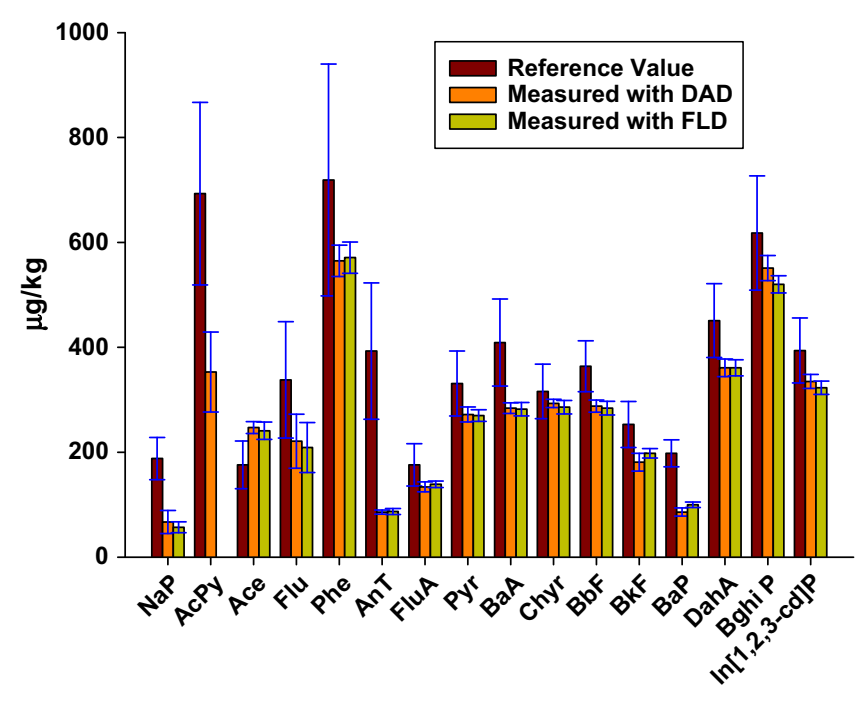

Fig. 3. The results of the $16 \mathrm{PAHs}$ in certified reference materials (CRMs).

typical chromatograms were produced as shown in Fig. 2. The regression equations, regression coefficients $\left(R^{2}\right)$, and detection limits (DL) are presented in Table 3. The results show that the relationship between peak area and PAH concentration was linear in the range $0.5-800.0 \mu \mathrm{g} \mathrm{L}^{-1}$ for $\mathrm{NaP}$ and $0.125-800.0 \mu \mathrm{g} \mathrm{L}^{-1}$ for the other 14 PAHs with FLD; in the case of DAD the linear concentration range was narrower and the relationship between peak area and $\mathrm{PAH}$ concentration was linear in the range 25.0$800.0 \mu \mathrm{g} \mathrm{L}^{-1}$ for Flu, Bghi P and BaP, $10.0-800.0 \mu \mathrm{g} \mathrm{L}^{-1}$ for FluA, $\mathrm{BbF}, \mathrm{BkF}$ and $\operatorname{In}[1,2,3-\mathrm{cd}] \mathrm{P}$, and $5.0-800.0 \mu \mathrm{g} \mathrm{L}^{-1}$ for the other 9 PAHs. All PAHs showed good linearity $\left(R^{2}>0.999\right)$ within the linear concentration range. The detection limits calculated with a signal to noise ratio of three (IUPAC criterion) were $0.01-0.1 \mu \mathrm{g} \mathrm{L}^{-1}$ for the 15 PAHs with FLD and 1.0-9.5 $\mu \mathrm{g} \mathrm{L}^{-1}$ for 16 PAHs with DAD. The precision represented as RSD from 7 replicate analyses of $25.0 \mu \mathrm{g} \mathrm{L}^{-1}$ PAHs was less than 5\% with DAD and 3\% with FLD. Our method was more sensitive than those previously reported for assaying the 16 PAHs in soils (Table S2, SD). For example, it was at least 20 times more sensitive than the HPLC-UV method (Sun et al., 1998), 4-5 times more sensitive than the HPLC-FLD method (Li et al., 2008), and comparable to the HPLC-FLD-UV method (Rao et al., 2007). Furthermore, the proposed method used DAD for PAH identity confirmation, which was more accurate for qualitative analysis of the 16 PAHs than the methods that use only RT for identify confirmation (Kuosmanen et al., 2003; Sun et al., 1998; Rao et al., 2007; Li et al., 2008).

\subsection{Recovery of the method}

The accuracy of the proposed method was tested using recovery experiments with soil samples spiked with three different concentrations $\left(37.5,75.0,115.5 \mu \mathrm{g} \mathrm{L}^{-1}\right)$ of standard PAH solution. The results are given in Table 4 and show that the average recovery varied between $61 \%$ and $96 \%$, except for NaP. The recovery of the lightest PAHs, such as NaP was lower $(<40 \%)$, possibly due to their distinct volatility loss during the evaporation process. Numerous studies have shown that the lightest PAHs are quite sensitive to the evaporation step (Kicinski et al., 1989; Bouzige et al., 1999).

\subsection{Validation of the method}

The applicability and reliability of the proposed HPLC-FLD-DAD method were tested by analyzing a certified reference material
(CRM 104) according to the procedures described in the proposed method. The results are shown in Fig. 3. The PAH concentrations measured using the proposed HPLC-FLD-DAD method are in the range of the reference values which were obtained by US EPA $8100,8270,8310$ or equivalent methods. Moreover, the concentrations of the PAHs quantified with FLD and DAD were consistent. Therefore, it is concluded that the proposed method is reliable.

\section{Conclusions}

An HPLC-FLD-DAD method was developed for determination of 16 PAHs in soil. By optimizing the mobile phase gradient elution program and the FLD wavelength switching program, we achieved complete baseline separation of 16 PAHs. DAD provided the match with the UV spectra of the 16 PAHs which was then used for peak identification and peak purity checks. Under optimal conditions, the detection limit was in the range of $1.0-9.5 \mu \mathrm{g} \mathrm{L}^{-1}$ for $16 \mathrm{PAHs}$ with DAD and 0.01-0.1 $\mu \mathrm{g} \mathrm{L}^{-1}$ for 15 PAHs with FLD. However, acenaphthylene (AcPy), which does not have fluorescence, cannot be easily quantified by FLD. As long as the concentration of samples above the detection limit of DAD, the specific spectra of all the 16 PAHs can be obtained from DAD, which contributed to confirm the identification of PAH components from complex samples and to quantify 16 PAHs at each specific wavelength. In case the concentration of samples is below the detection limit of DAD, the PAH components will only be determined by FLD which identifies the analytes only by their retention time. Therefore, the proposed method has the advantage of both FLD and DAD. Validation of the proposed method using a certified reference soil gave satisfactory results. We therefore conclude that the proposed HPLC-FLDDAD method is reliable for the determination low level PAHs in soils. In addition, with slight modifications the proposed method may be used for analysis of low level PAHs in other environmental samples such as sediments and waters.

\section{Acknowledgements}

This work was supported by grants from the Knowledge Innovation Program of the Chinese Academy of Sciences (Project Nos. KZCX2 -YW-Q02-02 and KZCX2-YW-BR-19) and the National Environmental Protection Public Welfare Research Fund (Project No. 201009032).

\section{Appendix A. Supplementary material}

Supplementary data associated with this article can be found, in the online version, at http://dx.doi.org/10.1016/j.chemosphere. 2013.03.035.

\section{References}

Bouzige, M., Pichon, V., Hennion, M.C., 1999. Class-selective immunosorbent for trace-level determination of polycyclic aromatic hydrocarbons in complex sample matrices, used in off-line program or on-line coupled with liquid chromatography/fluorescence and diode array detections in series. Environ. Sci. Technol. 33, 1916-1925.

Buco, S., Moragues, M., Doumenq, P., Noor, A., Mille, G., 2004. Analysis of polycyclic aromatic hydrocarbons in contaminated soil by Curie point pyrolysis coupled to gas chromatography-mass spectrometry, an alternative to conventional methods. J. Chromatogr., A 1026, 223-229.

Chen, Z.L., Megharaj, M., Naidu, R., 2002. Comparison of adsorbents for on-line solid-phase extraction of polycyclic aromatic hydrocarbons before liquid chromatography with UV detection. Chromatographia 56, 105-108.

Criado, A. Cárdenas, S., Gallego, M., Valcárcel, M., 2004. Direct automatic screening of soils for polycyclic aromatic hydrocarbons based on microwave-assisted extraction/fluorescence detection and on-line liquid chromatographic confirmation. J. Chromatogr., A 1050, 111-118.

Danyi, S., Brosea, F., Brasseur, C., Schneiderb, Y.J., Larondelle, Y., Pussemier, L., Robbens, J., Saeger, S.D., Maghuin-Rogister, G., Scippoa, M.L., 2009. Analysis of EU priority polycyclic aromatic hydrocarbons in food supplements using high 
1016

Y. Huang et al./Chemosphere 92 (2013) 1010-1016

performance liquid chromatography coupled to an ultraviolet, diode array or fluorescence detector. Anal. Chin. Anta 633, 293-299.

Ene, A., Bogdevich, O., Sion, A., Spans, T., 2012. Determination of polycyclic aromatic hydrocarbons by gas chromatography-mass spectrometry in soils from southeastern Romania. Microchem. J. 100, 36-41.

Jones, K.C., 1991. Contaminant trends in soils and crops. Environ. Pollute. 69, 311.

Kicinski, H.G., Adamek, S., Kettrup, A., 1989. Trace enrichment and HPLC analysis of polycyclic aromatic hydrocarbons in environmental samples, using solid phase extraction in connection with UV/Vis diode-array and fluorescence detection. Chromatographic 28, 203-208.

Kuosmanen, K., Hyötyläinen, T., Hartonen, K., Riekkola, M.L., 2003. Analysis of polycyclic aromatic hydrocarbons in soil and sediment with on-line coupled pressurised hot water extraction, hollow fibre microporous membrane liquidliquid extraction and gas chromatography. Analyst 128, 434-439.

Li, C.F., Wong, J.W.C., Hie, C.W., Choir, M.M.F., 2008. On-line flow injection-cloud point preconcentration of polycyclic aromatic hydrocarbons coupled with highperformance liquid chromatography. J. Chromatogr., A 1214, 11-16.

Lem, L.W., Okouchi, Y., Takeuchi, T., 2007. On-Line preconcentration of trace carcinogenic polycyclic aromatic hydrocarbons (DAHs) in microcolumn liquid chromatography via large volume injection. Talanta 72, 1600-1608.

Lu, H., Zhu, L.Z., Chen, S.G., 2008. Pollution level, phase distribution and health risk of polycyclic aromatic hydrocarbons in indoor air at public places of hangzhou, China. Environ. Pollute. 152, 569-575.

Ma, L.L., Chu, S.G., Wang, X.T., Ching, H.X., Lu, X.F., Xu, X.B., 2005. Polycyclic aromatic hydrocarbons in the surface soils from outskirts of Beijing, China. Chemosphere 58, 1355-1363.

Miègea, C., Dugay, J., Hennion, M.C., 2003. Optimization, validation and comparison of various extraction techniques for the trace determination of polycyclic aromatic hydrocarbons in sewage sludge by liquid chromatography coupled to diode-array and fluorescence detection. J. Chromatogr., A 995, 87-97.
Ozcan, S., Tor, A., Aydin, M.E., 2009. Determination of polycyclic aromatic hydrocarbons in soil by miniaturized ultrasonic extraction and gas chromatography-mass selective detection. Clean 37, 811-817.

Roo, Z., Li, S., He, M., Sur, J., 2007. Determination of 16 polycyclic aromatic hydrocarbons in soil by high performance liquid chromatography with fluorescence and ultraviolet detection. Chin. J. Anal. Chem. 35, 954-958.

Sikalos, T.I., Paleologos, E.K., 2005. Cloud point extraction coupled with microwave or ultrasonic assisted back extraction as a preconcentration step prior to gas chromatography. Anal. Chem. 77, 2544-2549.

Sun, F.S., Littlejohnb, D., Gibson, M.D., 1998. Ultrasonication extraction and solid phase extraction clean-up for determination of US EPA 16 priority pollutant polycyclic aromatic hydrocarbons in soils by reversed-phase liquid chromatography with ultraviolet absorption detection. Anal. Shim. Acts 364, 1-11.

Wang, Y.H., Zhang, J., Ding, Y.C., Zhou, J., Ni, L.X., Sun, C., 2009. Quantitative determination of 16 polycyclic aromatic hydrocarbons in soil samples using solid-phase microextraction. J. Sep. Sci. 32, 3951-3957.

White, K.L., 1986. An overview of immunotoxicology and carcinogenic polycyclic aromatic-hydrocarbons. J. Environ. Sci. Health 4, 163-202.

Wilcke, W., 2000. Polycyclic aromatichydrocarbons (DAHs) in soil - a review. J. Plant Nut. Soil Sci. 163, 229-248.

Yang, F., Long, Y.M., Chen, R., Chen, C.Y., Pan, D., Zhang, Q.L., Cai, Q.Y., Yo, S.Z., 2011. Ultrasonication extraction coupled with magnetic solid-phase clean-up for the determination of polycyclic aromatic hydrocarbons in soils by highperformance liquid chromatography. J. Sep. Sci. 34, 716-723.

Chang, W.H., Wei, C.H., Feng, C.H., Yu, Z., Ken, M., Yon, B., Peng, P.G., Pu, J.M., 2011. Distribution and health-risk of polycyclic aromatic hydrocarbons in soils at a coking plant. J. Environ. Mont. 13, 3429-3436.

Zuazagoitia, D., Millán, E., Garcia-Arrona, R., 2009. Comparison of two extraction methods for the determination of polycyclic aromatic hydrocarbons in surface soils using headspace SPME with GC-FID. J. Chromatogr. Sci. 47, 97-102. 